\title{
A quorum sensing-based in vivo expression system and its application in multivalent bacterial vaccine
}

Teng Chu ${ }^{1}$, Chunshan $\mathrm{Ni}^{1}$, Lingzhi Zhang ${ }^{1}$, Qiyao Wang ${ }^{1}$, Jingfan Xiao ${ }^{1}$, Yuanxing Zhang ${ }^{1,2}$ and Qin Liu ${ }^{1,2^{*}}$

\begin{abstract}
Background: Delivery of antigens by live bacterial carriers can elicit effective humoral and cellular responses and may be an attractive strategy for live bacterial vaccine production through introduction of a vector that expresses an exogenous protective antigen. To overcome the instability and metabolic burden associated with plasmid introduction, alternative strategies, such as the use of in vivo-inducible promoters, have been proposed. However, screening an ideal in vivo-activated promoter with high efficiency and low leak expression in a particular strain poses great challenges to many researchers.
\end{abstract}

Results: In this work, we constructed an in vivo antigen-expressing vector suitable for Edwardsiella tarda, an enteric Gram-negative invasive intracellular pathogen of both animals and humans. By combining quorum sensing genes from Vibrio fischeri with iron uptake regulons, a synthetic binary regulation system (ironQS) for E. tarda was designed. In vitro expression assay demonstrated that the ironQS system is only initiated in the absence of $\mathrm{Fe}^{2+}$ in the medium when the cell density reaches its threshold. The ironQS system was further confirmed in vivo to present an in vivo-triggered and cell density-dependent expression pattern in larvae and adult zebrafish. A recombinant E. tarda vector vaccine candidate WED(ironQS-G) was established by introducing gapA34, which encodes the protective antigen glyceraldehyde-3-phosphate dehydrogenase (GAPDH) from the fish pathogen Aeromonas hydrophila LSA34 into ironQS system, and the immune protection afforded by this vaccine was assessed in turbot (Scophtalmus maximus). Most of the vaccinated fish survived under the challenge with A. hydrophila LSA34 (RPS $=67.0 \%$ ) or E. tarda EIB202 (RPS $=72.3 \%$ ).

Conclusions: Quorum sensing system has been extensively used in various gene structures in synthetic biology as a well-functioning and population-dependent gene circuit. In this work, the in vivo expression system, ironQS, maintained the high expression efficiency of the quorum sensing circuit and achieved excellent expression regulation of the Fur box. The ironQS system has great potential in applications requiring in vivo protein expression, such as vector vaccines. Considering its high compatibility, ironQS system could function as a universal expression platform for a variety of bacterial hosts.

Keywords: Bacterial vector vaccine, Edwardsiella tarda, Iron uptake system, In vivo expression, Quorum sensing

\footnotetext{
*Correspondence: qinliu@ecust.edu.cn

${ }^{1}$ State Key Laboratory of Bioreactor Engineering, East China University of Science and Technology, 130 Meilong Road, Shanghai 200237, China

${ }^{2}$ Shanghai Collaborative Innovation Center for Biomanufacturing, Shanghai 200237, China
} 


\section{Background}

Vaccination constitutes the most cost-effective tool for prophylaxis of infectious diseases, and the use of bacterial carriers is probably one of the most successful strategies ever developed to deliver vaccine antigens [1]. The efficacy of a live bacterial vector vaccine rests in its ability to present sufficient antigens to the host immune system and initiate the desired protective immune response [2]. Selecting an appropriate expression strategy to optimize the production of the recombinant antigen is one of the most important issues relevant to the use of bacterial strains as vaccine carriers [3]. To date, certain strategies have been developed to achieve stable heterologous gene expression in vaccine vectors. Application of low copy number vectors and modification by introducing a balanced lethal system can improve the stability and safety of vaccines [4-6]. However, expression levels may be insufficient to stimulate the desired responses [7]. High copy number vectors achieve sufficient antigen expression but can cause over-attenuation of the carrier and lack of immunogenicity [8]. To circumvent these problems, optimal and controllable expression, such as with the use of in vivo-activated expression systems, is a crucial step in multivalent vaccine design.

In vivo environmental stimuli are represented by aggregates of unique signals, including anaerobic conditions [9], oxidizing agent availability [10], and low iron concentrations [11]. Several in vivo-inducible promoters that respond to these signals have been previously investigated [12]. For example, iron is often bound to metalchelating proteins in vivo, making it extremely limited in hosts [13]. Thus, lack of free iron is an important in vivo environmental signal [14]. To survive from the iron limited conditions of their hosts, bacteria have evolved several iron uptake, storage, and metabolism systems to obtain adequate iron in such an environment [15]. The promoter from iron-uptake regulon is strongly repressed in iron-rich conditions by Fur, a typical ferric uptake regulator protein, but fully de-repressed in the absence of iron [16]. A 19-bp inverted repeat consensus sequence, Fur box, which is known as the binding site of the Fur protein complexing with ferrous irons, is the control core of iron-responsive promoters in bacteria [17]. When iron is abundant, Fur protein forms a complex with ferrous iron and blocks the entry of RNA polymerase by binding to the Fur box in the relevant promoter area [18]. In our previous work, several ironregulated promoters and Fur boxes were applied to build in vivo-inducible regulation circuits for antigen expression, bacterial ghost preparation, and toxic protein synthesis [19-21].

Quorum sensing (QS) is a system that bacteria release and respond to membrane-penetrating molecules called autoinducers to regulate their gene expressions according to cell density [22]. This system allows bacteria to monitor a variety of processes, including competence, bioluminescence, virulence factor secretion, biofilm formation, and sporulation [23]. The first quorum sensing circuit was discovered in the bioluminescent marine bacterium Vibrio fischeri [24]. Two proteins, LuxI and LuxR, are essential for the quorum-sensing control of bioluminescence in $V$. fischeri. LuxI is the autoinducer synthase that produces an AHL, N-(3-oxohexanoyl)homoserine lactone [25]. LuxR is the cytoplasmic receptor of the autoinducer and the transcriptional activator of the luciferase luxICDABE operon [26,27]. At low cell density, the luxI gene is transcribed at a low basal level. As the culture grows and autoinducers accumulate to a specific threshold, cytoplasmic LuxR proteins combine with autoinducers and bind to the luxICDABE promoters [28]. Given that the expression of $\operatorname{luxI}$ is also activated by the autoinducer-bound LuxR, this autoinduction positive feedback loop is presumed to enforce synchrony as the cell population switches from low cell density mode to high cell density quorum sensing mode [29]. Quorum sensing systems have been widely used in various gene circuits in synthetic biology, serving as oscillator [30], amplifier [31] and so on [32-34], because of their high expression efficacy and cell density regulated characteristic. However, although a number of quorum sensing systems participate in various synthetic architectures; very few have been designed for bacterial vector vaccine.

In this work, several in vivo expression systems, including ironQS1-4, were developed for potential application in multivalent bacterial vaccine based on quorum sensing system of $V$. fischeri. To activate the quorum sensing system in vivo, iron starvation of the in vivo environment was utilized to adopt different strategies, including iron-regulated promoter substitution and iron-regulated regulator introduction. The screened ironQS system was demonstrated to be only initiated in iron-limited medium during in vitro expression assay when the cell density reached a threshold. The ironQS system was also well regulated by the iron signals and cell density in the subsequent in vivo expression assay. Furthermore, a protective antigen, glyceraldehyde-3-phosphate dehydrogenase (GAPDH) from the important fish pathogen Aeromonas hydrophila, was introduced to the ironQS system. The immune protection of the resultant recombinant $E$. tarda vector vaccine candidates was evaluated in turbot.

\section{Results and discussion}

\section{Construction of quorum sensing-based in vivo expression systems}

The quorum sensing components including $\operatorname{luxI}$, luxR and their respective promoters were cloned from $V$. fischeri. A 
reporter gene named katushka, which encoded a far-red fluorescent protein, was used to measure the expression efficiency of our systems. First, a basal quorum sensing regulated expression circuit was constructed through sequentially inserting a RBS component and a reporter gene katushka behind the intact QS regulon. A stable plasmid pUTat [35] containing this circuit was named pQS (Figure 1A), and four quorum sensing-based in vivo expression systems ironQS1-4 were designed on this basis (Figure 1B). The ironQS1 was a pQS derivative in which the original $\mathrm{P}_{l u x R}$ promoter was substituted for a strict low-iron-triggered promoter $\mathrm{P}_{v i u A}$ [35], so that ironQS1 would not express Katushka protein in ironrich culture medium. IronQS2 was constructed by inserting a standard Fur box sequence into the -10 region of luxI promoter, and ironQS3 and ironQS4 were the derivatives of ironQS2 with additional Fur boxes inserted striving for higher strictness. In culture medium, bacterial Fur proteins complexing with ferrous irons would bind to the Fur box sites to prevent transcriptional process of RNA polymerases, while in in vivo low-iron situation, ironQS2-4 systems would be de-repressed and started to produce interest proteins because of the dissociation of Fur proteins.

To evaluate the regulation and expression performances of four candidate systems, E. tarda recombinants harboring ironQS1-4 plasmids were cultured in both ironlimited and iron-rich media. Samples were adjusted to $\mathrm{OD}_{600}$ (optical density at $600 \mathrm{~nm}$ ) $=1$ to measure the red fluorescence emitted by Katushka. As seen in Figure 1C, in iron-limited environment, all four candidates showed strong fluorescence intensities with similar RFVs (relative fluorescence values) to that of the control system pQS. This was evidence that insertion of Fur box exerted no effects on expression efficiency. However, in iron-rich environment, their performances were divergent. IronQS2 presented the lowest leak expression level in iron-rich environment, while ironQS1 system showed the reduced expression strictness by the substitution of promoter $\mathrm{P}_{l u x R}$ by $\mathrm{P}_{v i u A}$. On the other hand, the additional Fur box in ironQS3 and ironQS4 did not play the desired effects to enhance their strictness. After comprehensive comparison of the four candidate expression systems, ironQS2, abbreviated to ironQS, was finally chosen as our desired expression system with the combined advantages of high induced expression level, high stringency and relatively simple genetic structure.

\section{In vitro expression of ironQS system}

To verify the iron-regulated and cell density-regulated performances of ironQS system, ironQS plasmid was transformed into E. tarda for the in vitro assay by adding $\mathrm{FeSO}_{4}$ or iron-chelator DP (2,2-dipyridyl) into the medium during cultivation. As showed in Figure 2A, there

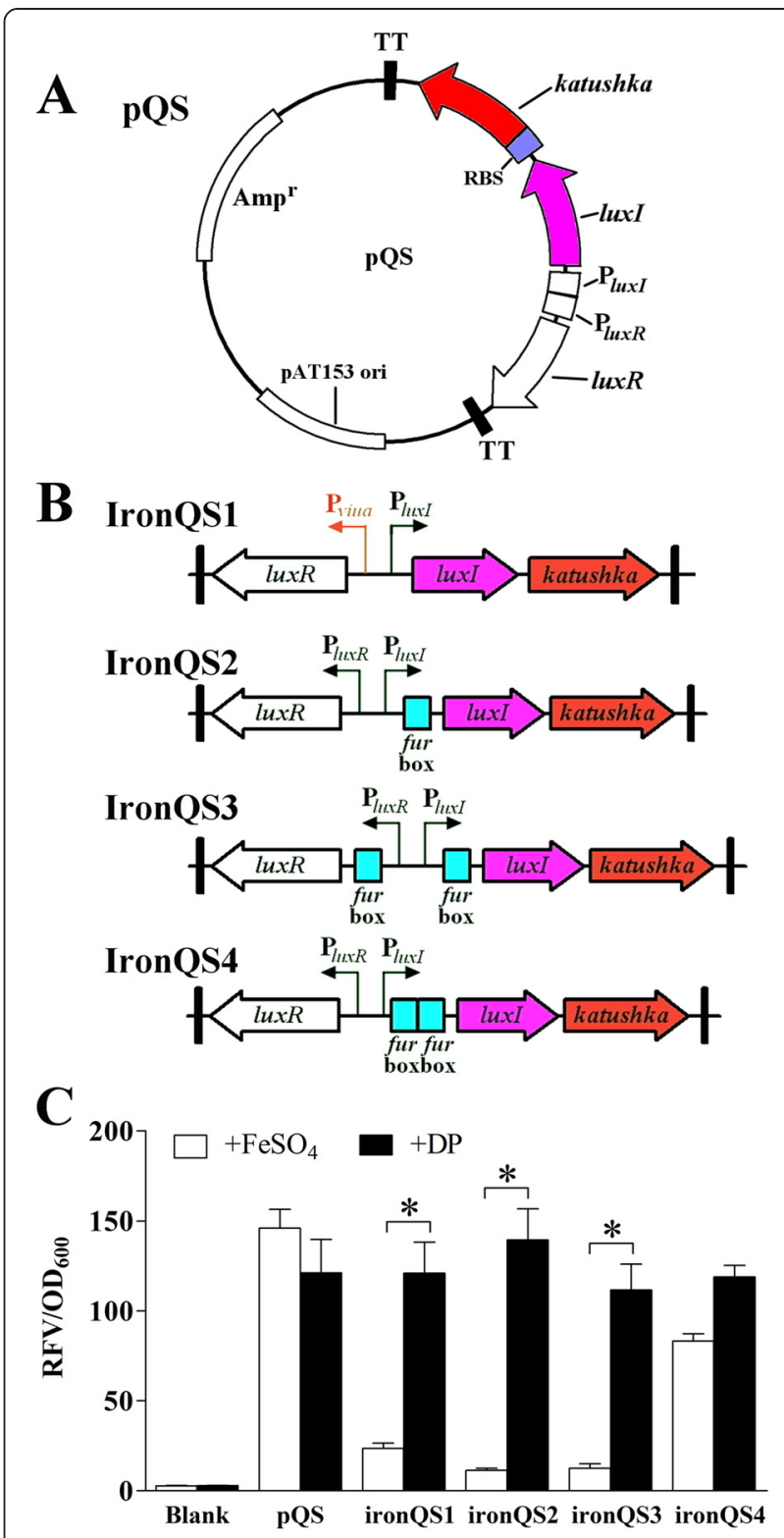

Figure 1 Construction and screening of ironQS systems. (A) Plasmid diagram of a basal cell density regulated expression plasmid pQS. $P_{\text {/ } u x /,}$ the promoter of luxl gene; $P_{\text {luxR, }}$ the promoter of luxR gene; $\pi$, transcription terminator; RBS, ribosome binding site; katushka, a reporter gene which product generates red fluorescence. (B) Linear structure illustrations of four quorum sensing-based in vivo expression systems ironQS1-4. (C) Relative fluorescence values of $E$. tarda strains containing ironQS1-4 after cultured in iron-rich and iron-limited media respectively. E. tarda loaded with $\mathrm{pQS}$ and blank strain were set as controls. * means a significant deviation.

was a low expression level of ironQS system in the presence of $\mathrm{Fe}^{2+}$. This indicated that Fur protein complexing with ferrous iron bond to the Fur box region and the QS system was remarkably repressed. On the contrary, ironQS was considerably activated in the absence of iron when $\mathrm{Fe}^{2+}$ in the medium was plundered by DP. 


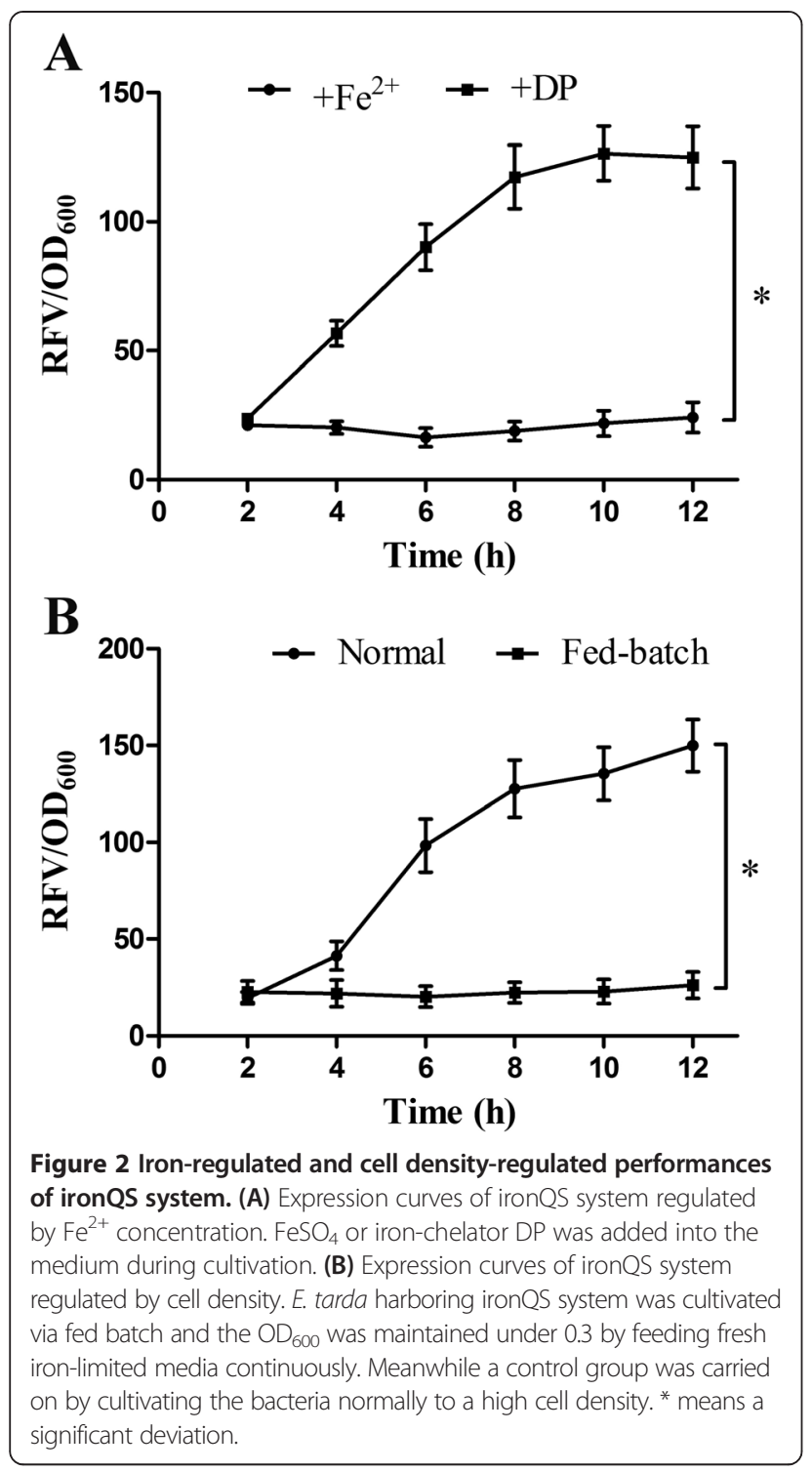

All these data confirmed that Fur box sequence could negatively control QS system by signaling to $\mathrm{Fe}^{2+}$ in the medium. Furthermore, to identify whether the cell density-regulated feature was still preserved in ironQS system, a fed-batch cultivation was conducted to control the bacterial cell density always under the threshold by continuously feeding fresh iron-free medium, and a control experiment was carried out by normally cultivating the bacteria to a high cell density. As shown in Figure 2B, RFV of ironQS strain in fed-batch model maintained at a low level, showing that the ironQS was always kept off even in iron-free medium when the cell density was below its threshold. Meanwhile, the RFV of control group rose continuously as the cell density exceeded threshold. These results suggested that ironQS system exhibited a precise iron and density dual-regulated expression feature by Fur box and quorum sensing circuit, respectively.

\section{In vivo expression of ironQS system}

In vivo condition is a typical iron-limited environment and a crucial site where bacterial vaccine plays its role. Although ironQS has been proved to be an efficient binary system responding to $\mathrm{Fe}^{2+}$ and cell density in vitro, the in vivo behaviors are particularly concerned. Since E. tarda is known as a facultative intracellular bacterium and able to persist and replicate within phagosomes of macrophages, it is reasonable to apply macrophages as a cell model for in vivo performance evaluation of ironQS. Suspensions of recombinant E. tarda WED containing ironQS were incubated in the macrophagelike cell J774A.1, and the fluorescence intensity was determined at regular time intervals with a fluorescence microscope. As shown in Figure 3A, cells infected by the bacterium showed only very low fluorescence in the first $2 \mathrm{~h}$ post-infection. During the whole period, the bacterial number maintained at $4.83 \pm 1.46$ per cell. Although WED was not likely to replicate in the macrophages, fluorescence intensity still became stronger gradually with the accumulation of autoinducers, indicating that ironQS system could sense the iron-limited signal in macrophage and unlock quorum sensing to express target protein. Meanwhile, the positive feedback of quorum sensing increased the expression of Katushka protein and enhanced the fluorescence intensity [36].

On the other hand, zebrafish larvae and adults were further applied as animal models to evaluate in vivo performance of ironQS. The larvae were infected by immersion with recombinant E. tarda WED strain, and the fluorescence in zebrafish larvae was determined at regular time intervals. As shown in Figure 3B, no obvious Katushka fluorescence signal was detected until $2 \mathrm{~h}$ post immersion and the fluorescence signals were mainly found in the gastrointestinal tract of fish. These results indicated that the ironQS system could function well in zebrafish larvae. Responding to the ironfree signal in larvae, ironQS was de-repressed after Fur protein dissociating from Fur box, and simultaneously, it was activated by locally accumulated autoinducers from an increasing bacterial population in vivo. For zebrafish adults, ironQS-loaded E. tarda strains were administrated by intraperitoneal injection, and then the in vivo expression pattern of ironQS was evaluated by Western blot. As showed in Figure 3C, ironQS was activated significantly after $12 \mathrm{~h}$ post injection upon in vivo propagation of the bacteria. This result indicated that attenuated E. tarda WED could colonize and persist in fish adult, in which the low iron concentration would trigger the expression of heterologous protein by ironQS. 


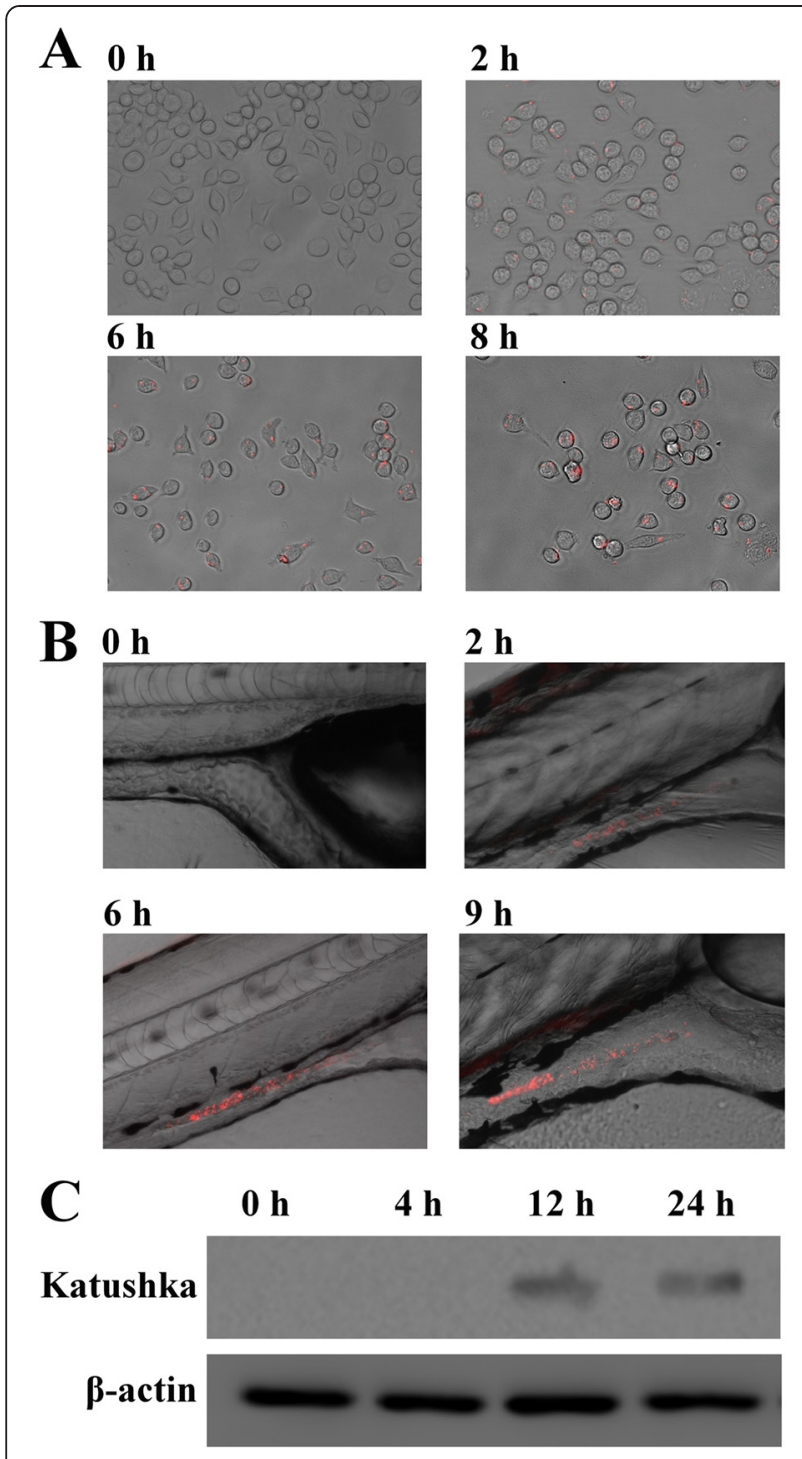

Figure 3 In vivo expressions of ironQS loaded $E$. tarda strains. (A) Expression of ironQS loaded strain in macrophages J774A.1. The infected cells were observed by inverted fluorescence microscope at different time intervals. (B) Expression of ironQS strain in zebrafish larvae. Zebrafish larvae were immersed in E. tarda(ironQS) suspension and observed by inverted fluorescence microscope at different time intervals. (C) Expression of ironQS strain in adult zebrafish. Zebrafish were intraperitoneally injected with $E$. tarda(ironQS) suspension. The internal organs were extracted at different time intervals and further analyzed by Western blot using the antibody specific to Katushka.

All the data confirmed that ironQS system could be effectively activated in vivo and the bacterial densitydependent expression feature, illustrated in vitro, was finely preserved. IronQS system maintained its dualregulated characteristics and made a good performance in in vivo environments such as in macrophage and zebrafish.

\section{Stress on bacterial growth and colonization}

Constitutive expression systems are often believed to produce significant metabolic burden on the host bacterium and thus may have negative effects on immune protection of bacterial vector vaccine. Therefore in vivo inducible expression system is highly recommended for bacterial vector vaccine design $[3,8]$. In this work, an in vivo inducible and cell density-dependent expression system ironQS was established. Compared with the original QS expression system, ironQS system exerted few effects on microbial in vitro growth and in vivo colonization. As showed in Figure 4A, when bacterial hosts containing $\mathrm{pQS}$, ironQS or blank plasmid pUTat were inoculated into fresh medium, WED(ironQS) showed a normal growth, which is similar to that of WED(pUTat), while the growth of WED(pQS) was obviously inhibited, especially during the early stage after inoculation. When three strains were injected into adult zebrafish, the colonization abilities were measured by counting viable bacterial numbers in zebrafish organs. As Figure 4B showed, a dramatic drop of viable bacterial numbers for WED(pQS) was found within zebrafish, while in vivo WED(ironQS) propagated significantly, similarly to the control strain WED(pUTat). These results suggested that the expression system ironQS, controlled by cell density and iron-limitation signal, brought about less growth stress on bacterial host and greatly contributed to the in vivo colonization and propagation of WED(ironQS), which would be a valuable attribute for a bacterial vector vaccine to evoke effective immune response in vivo.

\section{Expression of ironQS in broad bacterial hosts}

Adaptation to different bacterial hosts is an important aspect of a good heterologous expression system. IronQS system is composed of quorum sensing genes from $V$. fischeri and an artificial Fur box, and it is of paramount importance to work well in different bacterial hosts. Here, ironQS was transformed into gram-negative bacteria including genetic engineering strain Escherichia coli, fish-originated Vibrio anguillarum, and humanoriginated Salmonella typhimurium, as well as grampositive bacterium Staphylococcus aureus, respectively. In in vitro assays, ironQS in all the four strains presented no expression in normal iron-rich medium, and high expression in DP supplemented medium (Figure 5). According to the specificity of the quorum sensing system, LuxR receptor could only sense the autoinducer molecules produced by its homologous LuxI. Thus, quorum sensing circuit from $V$. fischeri had no interference with these bacterial hosts. Fur proteins in bacterial hosts could bind to or dissociate from the standard Fur box depending on iron concentration. The fine adaptability of ironQS system suggested that 

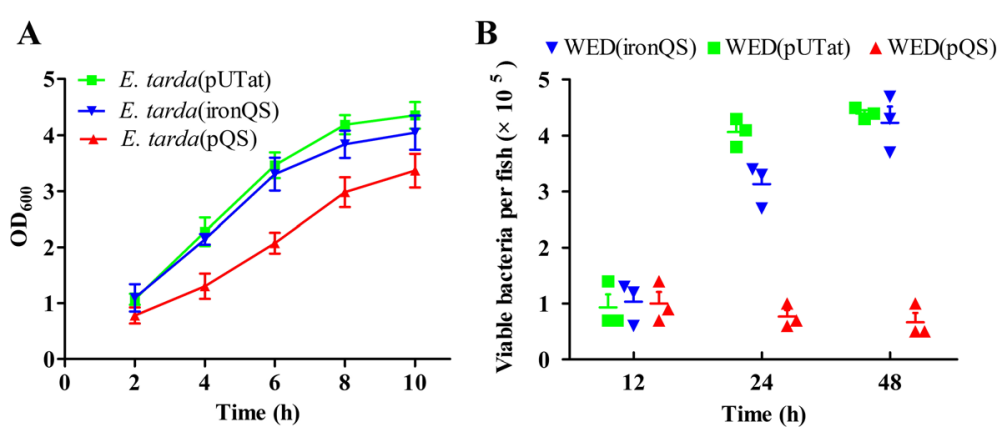

Figure 4 Metabolic burden of pQS and ironQS system in vitro and in vivo. (A) Growth curves of E. tarda loaded with pQS or ironQS plasmids cultivated in iron-rich medium, blank plasmid pUTat used as control. (B) Viable bacterial counts in each fish injected with these three strains $\left(5 \times 10^{4}\right.$ CFU per tail) at 12, 24 and $48 \mathrm{~h}$. Ten fish were set as a pool and three parallels were taken. At time points, 10 fish of each group were randomly picked, homogenized and the bacterial numbers were counted.

it might be potential to be used in a wide range of bacterial vaccine hosts.

\section{Application of ironQS in multivalent bacterial vaccine} GAPDH of $A$. hydrophila has been shown to be an effective protective antigen [37]. Edwardsiella tarda is an important facultative intracellular pathogen of both animals and humans, and its attenuated strain WED, constructed by in-frame deleting ese $B C D$ and $\operatorname{aro} C$, is an excellent bacterial vector for use in recombinant vaccine design [38]. The ironQS vector expressing heterologous antigen GAPDH was transformed into live attenuated bacterial vaccine $E$. tarda WED, and a multivalent bacterial vaccine candidate WED(ironQS-G) was constructed.

To investigate the potential application of WED (ironQS-G) as a multivalent vaccine, protection efficacy was evaluated in turbot, an important mariculture

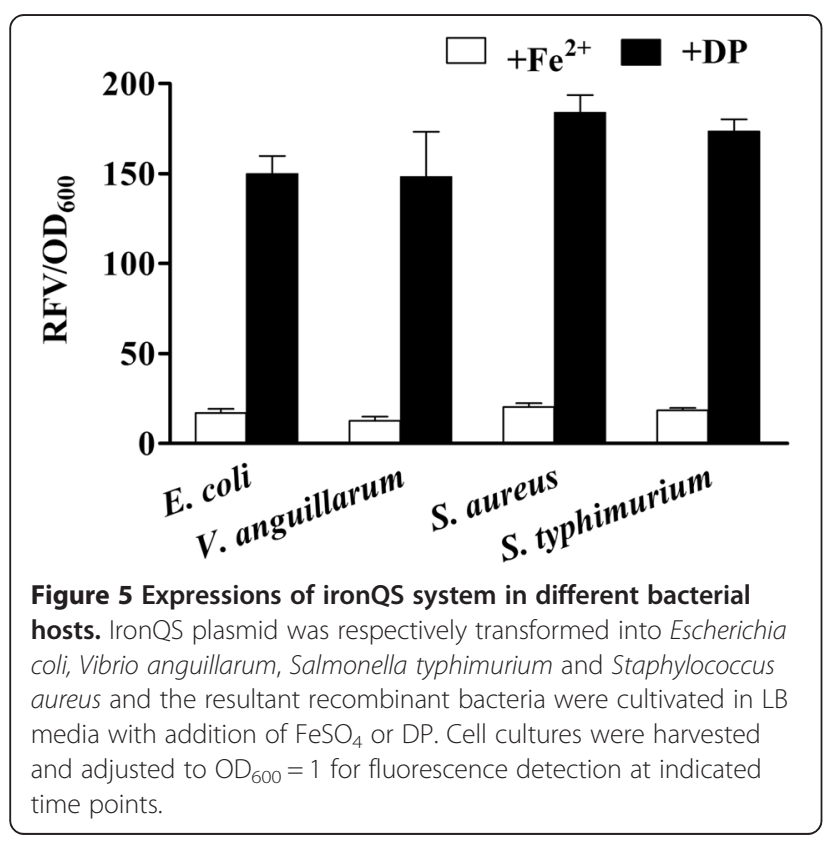

species in China. Vaccinated turbot were maintained for 30 days and then challenged by intraperitoneal injection with wild type $A$. hydrophila LSA34 or E. tarda EIB202. As shown in Table 1, WED(ironQS-G) showed significant multivalent protection against E. tarda EIB202 (RPS = 72.3\%) and A. hydrophila LSA34 (RPS $=67.0 \%$ ) over saline control. Meanwhile, the attenuated vector strain WED triggered significant protection against E. tarda EIB202 (RPS $=76.7 \%$ ), but only slight protection against $A$. hydrophila LSA34 (RPS $=13.9 \%)$. This confirmed that in vivo expression of GAPDH controlled by ironQS system conferred effective protection against experimental challenge with A. hydrophila LSA34, and also showed no adverse effect on the protection of E. tarda wildtype.

\section{Conclusions}

Quorum sensing system has been widely used in various gene structures in synthetic biology as a well-functioned and population-dependent gene circuit. Although these delicate genetic architectures fully embody the density dependent features and high expression efficiency, few of such systems have been designed and implemented in practical applications [39]. In this work, an efficient and finely-regulated in vivo expression system, ironQS, was designed by integrating Fur box with quorum sensing circuit of $V$. fischeri. Binding of the Fur-iron complex functioned as a lock that prevents gene transcription of quorum sensing circuit and then shuts down the entire expression system. However, given the extraordinary lack of free iron in vivo, Fur protein dissociated from Fur box and activated quorum sensing circuit. Experiments on in vitro simulation and zebrafish model confirmed that the ironQS system exhibits an in vivo-triggered and cell density-dependent expression pattern.

For practical application, we established a bacterial vector vaccine using the ironQS system as an antigen presenter. Several characteristics dominate the efficiency of a live bacterial vector vaccine: stability, infectivity and 
Table 1 Efficacy of vaccine candidate WED(ironQS-G) against E. tarda EIB202 and A. hydrophila LSA34 in turbot

\begin{tabular}{|c|c|c|c|c|c|c|c|}
\hline Immunogen & Vaccination dose & Challenge strain & Challenge dose (CFU/tail) & No. of fish & Mortality $^{\mathrm{a}}(\%)$ & RPS $^{\mathrm{b}}(\%)$ & Effect $^{c}$ \\
\hline \multirow[t]{2}{*}{ Saline } & $0.1 \mathrm{ml} /$ tail & EIB202 & $6 \times 10^{3}$ & $30 \times 3^{d}$ & $100 \pm 0^{e}$ & / & / \\
\hline & & LSA34 & $8 \times 10^{7}$ & $30 \times 3$ & $85 \pm 5.8$ & / & / \\
\hline \multirow[t]{2}{*}{ WED } & $10^{7} \mathrm{CFU} /$ tail & EIB202 & $6 \times 10^{3}$ & $30 \times 3$ & $23.3 \pm 4.7$ & $76.7 \pm 4.7$ & + \\
\hline & & LSA34 & $8 \times 10^{7}$ & $30 \times 3$ & $73.3 \pm 5.8$ & $13.9 \pm 6.8$ & - \\
\hline \multirow[t]{2}{*}{ WED(ironQS-G) } & $10^{7} \mathrm{CFU} /$ tail & EIB202 & $6 \times 10^{3}$ & $30 \times 3$ & $27.7 \pm 5.0$ & $72.3 \pm 5.0$ & + \\
\hline & & LSA34 & $8 \times 10^{7}$ & $30 \times 3$ & $28.0 \pm 7.5$ & $67.0 \pm 8.9$ & + \\
\hline
\end{tabular}

${ }^{a}$ The mortality was recorded for 4 weeks after challenge, and the observation of surviving fish was extended to 6 weeks.

${ }^{b}$ RPS is the relative percent survival, which is defined relative to the saline group. RPS $(\%)=(1-$ mortality of vaccinated fish / mortality of control fish) $\times 100$.

"If RPS $>60 \%$, it represents that the vaccine candidate showed protection against challenge, marked as " + ". If RPS $<30 \%$, it represents no protection against

challenge strain, marked as "-".

d " $\times 3$ " represents the vaccination and challenge experiments were done in three parallels.

"The " \pm " is standard deviation.

ability to present sufficient antigen [3]. How to improve antigen delivery efficiency without reducing vaccine stability and vitality has long challenged researchers. The use of plasmids in vector vaccines is often associated with instability of the recombinant phenotype and declining ability of colonization. In the ironQS system, several strategies were adopted to render it more suitable for use in a vector vaccine. First, as the main switch of the entire system, Fur box prevented unnecessary expression during in vitro cultivation, thereby alleviating the metabolic burden during cultivation and enhancing the ability of colonization of the bacterial host at early infection stages [40]. Second, thorough de-repression of the quorum sensing system completely restored the high expression efficiency of the system to guarantee sufficient antigen production in the host. Benefited from the population-dependent expression pattern of quorum sensing circuit, undesirable antigen protein expression was controlled to a minimum during the first stage of colonization because of the low cell density, until the bacterial population reached its threshold and then evoked multiple protective immune responses in fish host. Taken these results together, the ironQS system proposed in this work has great potential in designs of a stable and efficient bacterial vector vaccine.

The iron-dependent gene regulation mechanism has been well studied in different bacteria, and certain ironregulated promoters have been used to build in vivo inducible regulation circuits for controlled antigen expression $[19,35]$. Prior to designing a vector vaccine, a desirable iron-regulated promoter must be screened for the corresponding host because promoters borrowed from other host strains often function unsatisfactorily. This problem could be readily circumvented by introduction of the ironQS system. Although the various host strains used in this work possess their own QS systems and produce their own autoinducers, the LuxR receptors only identify their own autoinducers because these signal molecules vary in their acyl side chains $[22,23]$. Thus, no crosstalk or interference was observed between the native host QS and the heterologous ironQS because of the specificity of quorum sensing system. As well, the 19-bp consensus Fur box sequence is highly conserved [16]. Hence, the ironQS system displays high compatibility with a variety of bacterial hosts, and this plasmid forms a selfcontained and closed-loop expression circuit that is less dependent on its hosts. In fact, ironQS can function as a universal platform that is suitable for multiple hosts, rather than as a specific vector for E. tarda, in this work. This broad suitability suggests that, theoretically, any antigen protein can be loaded into the ironQS system and this system may be introduced into any bacterial host to prepare the desired vaccines simply and directly.

In summary, we established an in vivo programmed expression circuit that is de-repressed by iron-free signals in vivo and then programmed to produce heterologous proteins in a cell density-dependent manner in vivo. This artificial synthetic circuit might have potential use for in vivo applications including, but not limited to, bacterial vector vaccines.

\section{Methods}

\section{Bacterial strains and growth conditions}

Strains used in this study are listed in Table 2. E. coli strain was grown at $37^{\circ} \mathrm{C}$ in Luria-Bertani (LB) medium ( $1 \%$ tryptone, $0.5 \%$ yeast extract, $1 \% \mathrm{NaCl}$ ). Edwardsiella tarda, Aeromonas hydrophila, Vibrio anguillarum, Salmonella typhimurium and Staphylococcus aureus strains were grown at $30^{\circ} \mathrm{C}$ in $\mathrm{LB}$ medium. When required, the antibiotics (Sigma, USA) ampicillin (Amp) and colistin (Col) were added at corresponding final concentrations of $100 \mu \mathrm{g} / \mathrm{ml}$ and $12.5 \mu \mathrm{g} / \mathrm{ml}$, and $\mathrm{FeSO}_{4}(40 \mu \mathrm{M})$ or 2,2dipyridyl (DP, $200 \mu \mathrm{M}$ ) was added to create iron-rich or iron-limited condition [35].

\section{Plasmid construction}

General DNA operations were conducted following standard protocols. Automated DNA sequencing and primer 
Table 2 Strains and plasmids used in this study

\begin{tabular}{|c|c|c|}
\hline Strains and plasmids & Information & Source \\
\hline \multicolumn{3}{|l|}{ Strains } \\
\hline Vibrio fischeri MJ11 & Wild type, used for cloning of luxR/I quorum sensing system & MCCC \\
\hline Edwardsiella tarda EIB202 & Wild type, fish pathogen, broad range testing host & Our lab \\
\hline Edwardsiella tarda WED & $\begin{array}{l}\text { Mutant disrupted in type III secretion system and chorismic acid synthesis, a live } \\
\text { attenuated vaccine }\end{array}$ & Our lab \\
\hline Escherichia coli BL21 & General expressing strain, broad range testing host & Novagen \\
\hline Vibrio anguillarum MVM425 & Wild type, fish pathogen, broad range testing host & Our lab \\
\hline Staphylococcus aureus & Wild type, human pathogen, broad range testing host & Our lab \\
\hline Salmonella typhimurium & Wild type, human pathogen, broad range testing host & Our lab \\
\hline Aeromonas hydrophila LSA34 & Gene source of protective antigen GAPDH & Our lab \\
\hline \multicolumn{3}{|l|}{ Plasmids } \\
\hline pUTat & Expression vector, Amp ${ }^{r}$ & Our lab [35] \\
\hline pQS & $\begin{array}{l}\text { pUTat vector containing intact QS gene elements and a reporter gene co-transcribed } \\
\text { with luxl }\end{array}$ & This work \\
\hline ironQS1 & $\begin{array}{l}\text { pQS plasmid derivate in which the original } \mathrm{P}_{\text {luxR }} \text { promoter was substituted for a } \\
\text { low-iron-triggered promoter } \mathrm{P}_{\text {viuA }}\end{array}$ & This work \\
\hline ironQS2 & pQS plasmid derivate inserted with a standard Fur box into -10 region of lux/ promoter & This work \\
\hline ironQS3 & $\begin{array}{l}\text { PQS plasmid derivate inserted with two standard Fur boxes into }-10 \text { region of luxl and } \\
\text { luxR promoters }\end{array}$ & This work \\
\hline ironQS4 & $\begin{array}{l}\text { pQS plasmid derivate inserted with two continuous Fur box into }-10 \text { region of luxl } \\
\text { promoter }\end{array}$ & This work \\
\hline ironQS-G & ironQS plasmid in which reporter gene was substituted by gapA34 gene & This work \\
\hline
\end{tabular}

synthesis were completed by Life Technologies (Shanghai, China). Seamless cloning operations were carried out using ClonExpress II One Step Cloning Kit (Vazyme Co. Ltd., China) following standard procedures. The plasmids used or constructed in this work were listed in Table 1. First, a basal quorum sensing regulated expression plasmid $\mathrm{pQS}$ was constructed on the basis of pUTat expression vector by sequentially inserting a RBS sequence and a reporter gene katushka behind the intact QS regulon. The DNA fragments of pUTat, katushka and QS gene were respectively amplified with their primers that overlapping with each other (primers pUT-F/R for linear pUTat, katushka-F/R for gene katushka, QS-F/R for quorum sensing circuit). By seamless cloning operation, the fragments were spliced to form the intact pQS plasmid. On the basis of $\mathrm{pQS}$, four quorum sensing-based in vivo expression plasmids ironQS1-4 were designed by replacing promoters or inserting Fur boxes into basal plasmid pQS. IronQS1 was constructed by replacing the original luxR promoter with an iron-regulated promoter $\mathrm{P}_{v i u A} \cdot \mathrm{P}_{\text {viuA }}$ was amplified from the genome of Vibrio anguillarum MVM425 using primers $\mathrm{P}_{v i u A}-\mathrm{F} / \mathrm{R}$ and the rest was amplified from $\mathrm{pQS}$ using ironQS1-F/R. These two fractions were then jointed through seamless cloning operation. IronQS2 was constructed by inserting a standard Fur box behind luxI promoter to block its transcription in ironrich condition. Using primers ironQS2-F/R, pQS plasmid was amplified, linearized and then self-ligated via seamless cloning to form ironQS2. To enhance this modification, another Fur box sequence was subsequently added behind luxR promoter to form ironQS3 using primers ironQS3-F/R. Similarly, in ironQS4, two continuous Fur box sequences were inserted behind luxI promoter for the purpose of improving iron regulation. IronQS4 was constructed by amplifying and linearizing $\mathrm{pQS}$ using primers ironQS4-F/R and make it self-ligated via seamless cloning operation. For construction of vaccine vector ironQS-G, the gene gapA34 encoding glyceraldehyde3-phosphate dehydrogenase from Aeromonas hydrophila LSA34 was amplified using primers gapA-F/R, and ironQS was linearized to remove the reporter gene using primers ironQSG-F/R. These two fragments were assembled together by seamless cloning operation. Primers used in this work were listed in Table 3.

\section{Expression assay of ironQS strains in vitro}

Overnight bacterial cultures were inoculated $(1: 1000, \mathrm{v} / \mathrm{v})$ into fresh $\mathrm{LB}$ medium with antibiotics and $\mathrm{FeSO}_{4}$. For fed-batch cultivation, fresh LB medium with antibiotics (and $\mathrm{DP}$ or $\mathrm{FeSO}_{4}$ if needed) was continuously added into culture vessel to keep $\mathrm{OD}_{600}$ under 0.3 all the time, below the cell density threshold to activate the established pQS system in this work. At different time intervals, each culture sample was centrifuged at $8000 \times g$ for $5 \mathrm{~min}$, and the 
Table 3 Primers used in this study

\begin{tabular}{|c|c|}
\hline Primers & Sequence $\left(5^{\prime}-3^{\prime}\right)$ \\
\hline pUT-F & CAAGCTGGGTCACAGCTGAGTCGACCTGCAGCCAAGCTT \\
\hline pUT-R & CCCATACTTTAAAAATTAAGGATCCCCGGGAATTC \\
\hline QS-F & CCCGGGGATCCTTAATTITTAAAGTATGGGC \\
\hline QS-R & CGCCCACCATATAATTTCCTTTAATTAATTTAAGACTGC \\
\hline katushka-F & TTAAAGGAAATTATATGGTGGGCGAGGATAGCGTGC \\
\hline katushka-R & GGCTGCAGGTCGACTCAGCTGTGACCCAGCTTGCTC \\
\hline$P_{\text {viuA }}-\mathrm{F}$ & GTITTCATATG AATTTCTCCTTAACTCTA \\
\hline$P_{\text {viuA }}-R$ & GATAAAGAGATGCATATGCGACTGAGCGATGTAAAAC \\
\hline ironQS1-F & ATCGCTCAGTCGCATATGCATCTCTTTATCC \\
\hline ironQS1-R & GAAATTCATATGAAAAACATAAATGCCGAC \\
\hline ironQS2-F & GATAATGATAATCATTATCAATAAACGCAAGGGAG \\
\hline ironQS2-R & GATAATGATTATCATTATCCGACTATAACAAACCATTTTC \\
\hline ironQS3-F & GATAATGATAATCATTATCACCTATTGTTTGTCGC \\
\hline ironQS3-R & GATAATGATTATCATTATCAAGGATAAAGAGATGC \\
\hline ironQS4-F & $\begin{array}{l}\text { GATAATGATAATCATTATCGATAATGATAATCATTATCAAT } \\
\text { AAACGCAAGGGAGG }\end{array}$ \\
\hline ironQS4-R & $\begin{array}{l}\text { GATAATGATTATCATTATCGATAATGATTATCATTATCCGAC } \\
\text { TATAACAAACCATIITC }\end{array}$ \\
\hline gapA-F & AAATTAATTAAAGGAAATTATATGACTATCAAAGTAGG \\
\hline gapA-R & GCTTGGCTGCAGGTCGACTTACTTAGAGATGTGAG \\
\hline ironQSG-F & CACATCTCTAAGTAAGTCGACCTGCAGCCAAGC \\
\hline ironQSG-R & ACTTTGATAGTCATATAATTTCCTITAATTAATTTAAGACTGC \\
\hline
\end{tabular}

harvested cells were washed with PBS for three times, and resuspended in PBS. To measure the expression level per unit of bacteria, all the samples were diluted to the same $\mathrm{OD}_{600}$ value $\left(\mathrm{OD}_{600}=1.0\right)$ and $100 \mu \mathrm{l}$ of each cell suspension was added into a 96-well flat-bottom polystyrene plate (Costar, USA) for fluorescence detection, using a fluorescence microplate reader (Molecular Devices SpectraMax M5, USA). For Katushka protein detection, excitation wavelength was set at $588 \mathrm{~nm}$ and emission at $633 \mathrm{~nm}$.

\section{Expression assay of ironQS strains in macrophage}

The mouse macrophage-like cell line J774A.1 was purchased from American Type Culture Collection (ATCC). Cells were cultured at $37^{\circ} \mathrm{C}$ in 24-well plate in DMEM (Gibco BRL, Eggenstein, Germany) supplemented with $10 \%$ fetal bovine serum (Gibco BRL, Eggenstein, Germany) in a humidified $5 \% \mathrm{CO}_{2}$ atmosphere to obtain monolayers and the cell number was controlled at $3 \times 10^{5}$ cells per well. Overnight bacterial cultures of $E$. tarda(ironQS) were inoculated (1:1000, v/v) into fresh LB medium with antibiotics and $\mathrm{FeSO}_{4}$, and were harvested and suspended in PBS. For each sample, $500 \mu \mathrm{l}$ of bacterial suspension was added into the well at a moi ratio of 10:1, and incubated at $35^{\circ} \mathrm{C}$ for $2 \mathrm{~h}$. Macrophages were then washed for three times and DMEM containing gentamicin $(100 \mu \mathrm{g} / \mathrm{ml})$ was added to inhibit the growth of remaining extracellular bacteria. At different time intervals, samples were observed by inverted fluorescence microscope (Leica DMI3000B, Germany). At the same time, the culture medium of corresponding wells was removed and washed with PBS for three times. Then the plate was added $500 \mu \mathrm{l}$ Triton-100 (1\%) per well and incubated in $35^{\circ} \mathrm{C}$ for $0.5 \mathrm{~h}$. In a series of dilution, the lysed suspensions were coated on antibiotic agar plates and the bacterial number within each cell was calculated.

\section{Expression assay of ironQS strains in zebrafish model}

Overnight bacterial cultures of E. tarda(ironQS) were inoculated $(1: 1000, \mathrm{v} / \mathrm{v})$ into fresh LB medium with antibiotics and $\mathrm{FeSO}_{4}$. The bacteria were incubated for $9 \mathrm{~h}$, harvested, and suspended in PBS. Zebrafish larvae at an age of the 6th to 8th day were immersed in cell suspension at a concentration of $10^{8}$ viable bacteria per $\mathrm{ml}$ for $2 \mathrm{~h}$ and then were washed and transferred into freshwater. At target time point, larvae were washed with PBS at least three times, anesthetized with $0.01 \%(\mathrm{w} / \mathrm{v})$ anesthetic MS-222 and observed with an inverted fluorescence microscope.

For zebrafish adults, bacterial cultures were treated as above. Healthy adult zebrafish with an average weight of $0.2 \mathrm{~g}$ were intraperitoneally injected with $E$. tarda (ironQS) suspension at the dose of $10^{5} \mathrm{CFU}$ (colony forming unit) per tail. At $4 \mathrm{~h}, 12 \mathrm{~h}$ and $24 \mathrm{~h}$ post injection, the internal organs of 20 fish from each group were surgically extracted. After homogenized and concentrated with ultrafiltration, the tissue samples were further analyzed by Western blot using the antibody specific to Katushka.

\section{Vaccination and challenge}

The recombinant plasmid ironQS-G was transformed into attenuated $E$. tarda vaccine strain WED to get WED(ironQS-G). This potential multivalent vaccine candidate WED(ironQS-G) was assayed for the immune protection in turbot. Attenuated E. tarda strain WED was used as the control. All the vaccination and challenge experiments were done in triplicate. For vaccination, turbot (Scophtalmus maximus) weighed about $10 \mathrm{~g}$ from an aquaculture farm in Shandong Province, China, were fed and acclimated for 30 days before experiment. Three turbot groups were intraperitoneally injected with sterilized saline $(0.1 \mathrm{ml}$ per tail), WED suspension $\left(10^{7} \mathrm{CFU}\right.$ per tail) and WED(ironQS-G) suspension $\left(10^{7} \mathrm{CFU}\right.$ per tail), respectively. The immunized fish were reared in aquaria supplied with a continuous flow of recycling water at $16-18^{\circ} \mathrm{C}$. For challenge, 4 weeks later, each immunized group was divided into 2 challenge subgroups which were intramuscularly injected with wild type $A$. hydrophila $\left(8 \times 10^{7} \mathrm{CFU}\right.$ per tail) and 
wild type E. tarda EIB202 $\left(6 \times 10^{3} \mathrm{CFU}\right.$ per tail), respectively. The mortality was recorded for 4 weeks after challenge, and the observation of surviving fish was extended to 6 weeks. The significant difference and the relative percent survival (RPS) were, respectively, calculated by using Fisher's exact test and according to the formula:

\author{
$\operatorname{RPS}(\%)$ \\ $=(1-$ mortality of vaccinated fish/mortality of control fish $)$ \\ $\times 100$
}

\begin{abstract}
Abbreviations
QS: Quorum sensing system; DP: 2,2-dipyridyl; RFV: Relative fluorescence value; GAPDH: Glyceraldehyde-3-phosphate dehydrogenase; OD: Optical density; CFU: Colony forming unit; RPS: Relative percent survival.
\end{abstract}

\section{Competing interests}

The authors declare that they have no competing interests.

\section{Authors' contributions}

TC and QL designed the QS and araQS systems. TC and JX analyzed the in vitro expression of araQS. TC, CN and LZ performed in vivo studies of araQS in macrophages and zebrafish. TC tested araQS's performances in broad bacterial hosts. TC and QW designed the vaccine and evaluated its protection efficacy in turbot. TC, QL and YZ wrote the manuscript. All authors read and approved the final manuscript.

\section{Acknowledgments}

This work was financially supported by grants from the National Natural Science Funds of China (31272700), National Key Technology Support Program of China (2012BAD17B02) and National High Technology Research and Development Program of China (2013AA093101).

\section{Received: 25 September 2014 Accepted: 19 February 2015}

\section{Published online: 18 March 2015}

\section{References}

1. Loessner H, Endmann A, Leschner S, Bauer H, Zelmer A, Zur Lage S, et al. Improving live attenuated bacterial carriers for vaccination and therapy. J Med Microbiol. 2008;298:21-6.

2. Spreng S, Dietrich G, Weidinger G. Rational design of Salmonella-based vaccination strategies. Methods. 2006;38:133-43.

3. Medina E, Guzmán CA. Use of live bacterial vaccine vectors for antigen delivery: potential and limitations. Vaccine. 2001;19:1573-80.

4. Tzschaschel BD, Klee SR, de Lorenzo V, Timmis KN, Guzman CA. Towards a vaccine candidate against Shigella dysenteriae 1: expression of the Shiga toxin B-subunit in an attenuated Shigella flexneri aroD carrier strain. Microb Pathog. 1996;21:277-88.

5. Galen JE, Nair J, Wang JY, Wasserman SS, Tanner MK, Sztein MB, et al. Optimization of plasmid maintenance in the attenuated live vector vaccine strain Salmonella typhi CVD 908-htrA. Infect Immun. 1999;67:6424-33.

6. Yan YJ, Mu W, Zhang LZ, Guan LY, Liu Q, Zhang YX. Asd-based balanced-lethal system in attenuated Edwardsiella tarda to express a heterologous antigen for a multivalent bacterial vaccine. Fish Shellfish Immunol. 2013;34:1188-94.

7. Cardenas L, Clements JD. Stability, immunogenicity and expression of foreign antigens in bacterial vaccine vectors. Vaccine. 1993;11:126-35.

8. Pappalardo F, Halling-Brown MD, Rapin N, Zhang P, Alemani D, Emerson A et al. ImmunoGrid, an integrative environment for large-scale simulation of the immune system for vaccine discovery, design and optimization. Brief Bioinform. 2009;10:330-40.

9. Salam MA, Katz J, Zhang P. Hajishengallis G, Michalek SM. Immunogenicity of Salmonella vector vaccines expressing SBR of Streptococcus mutans under the control of a T7-nirB (dual) promoter system. Vaccine. 2006;24:5003-15.
10. Christman MF, Morgan RW, Jacobson FS, Ames BN. Positive control of a regulon for defenses against oxidative stress and some heat-shock proteins in Salmonella typhimurium. Cell. 1985;41:753-62.

11. Ma JM, Xu JM, Guan LY, Hu TJ, Liu Q, Xiao JF, et al. Cell-penetrating peptides mediated protein cross-membrane delivery and its use in bacterial vector vaccine. Fish Shellfish Immunol. 2014;39:8-16.

12. Voigt CA. Genetic parts to program bacteria. Curr Opin Biotechnol. 2006;17:548-57.

13. Bury NR, Grosell M. Waterborne iron acquisition by a freshwater teleost fish, zebrafish Danio rerio. J Exp Biol. 2003;206:3529-35.

14. Payne SM. Iron acquisition in microbial pathogenesis. Trends Microbiol. 1993;1:66-9.

15. Santander J, Golden G, Wanda SY, Curtiss 3rd R. Fur-regulated iron uptake system of Edwardsiella ictaluri and its influence on pathogenesis and immunogenicity in the catfish host. Infect Immun. 2012;80:2689-703.

16. Baichoo N, Helmann JD. Recognition of DNA by Fur: a reinterpretation of the Fur box consensus sequence. J Bacteriol. 2002;184:5826-32.

17. Escolar L, Perez-Martin J, de Lorenzo V. Binding of the fur (ferric uptake regulator) repressor of Escherichia coli to arrays of the GATAAT sequence. J Mol Biol. 1998;283:537-47.

18. Stojiljkovic I, Baumler AJ, Hantke K. Fur regulon in gram-negative bacteria. Identification and characterization of new iron-regulated Escherichia coli genes by a fur titration assay. J Mol Biol. 1994;236:531-45.

19. Mu W, Guan LY, Yan YJ, Liu Q, Zhang YX. A novel in vivo inducible expression system in Edwardsiella tarda for potential application in bacterial polyvalence vaccine. Fish Shellfish Immunol. 2011;31:1097-105.

20. Guan LY, Mu W, Champeimont J, Wang QY, Wu HZ, Xiao JF, et al. Iron-regulated lysis of recombinant Escherichia coli in host releases protective antigen and confers biological containment. Infect Immun. 2011;79:2608-18.

21. Guan LY, Liu Q, Li C, Zhang YX. Development of a Fur-dependent and tightly regulated expression system in Escherichia coli for toxic protein synthesis. BMC Biotechnol. 2013;13:25.

22. Miller MB, Bassler BL. Quorum sensing in bacteria. Annu Rev Microbiol. 2001:55:165-99.

23. Bassler BL, Losick R. Bacterially speaking. Cell. 2006;125:237-46.

24. Ruby EG. Lessons from a cooperative, bacterial-animal association: the Vibrio fischeri-Euprymna scolopes light organ symbiosis. Annu Rev Microbiol. 1996;50:591-624

25. Eberhard A, Burlingame AL, Eberhard C, Kenyon GL, Nealson KH, Oppenheimer NJ. Structural identification of autoinducer of Photobacterium fischeri luciferase. Biochemistry. 1981;20:2444-9.

26. Engebrecht J, Silverman M. Identification of genes and gene products necessary for bacterial bioluminescence. Proc Natl Acad Sci U S A. 1984;81:4154-8.

27. Engebrecht J, Nealson K, Silverman M. Bacterial bioluminescence: isolation and genetic analysis of functions from Vibrio fischeri. Cell. 1983;32:773-81.

28. Stevens AM, Dolan KM, Greenberg EP. Synergistic binding of the Vibrio fischeri LuXR transcriptional activator domain and RNA polymerase to the lux promoter region. Proc Natl Acad Sci U S A. 1994:91:12619-23.

29. Ng WL, Bassler BL. Bacterial quorum-sensing network architectures. Annu Rev Genet. 2009:43:197-222.

30. Danino T, Mondragon-Palomino O, Tsimring L, Hasty J. A synchronized quorum of genetic clocks. Nature. 2010:463:326-30.

31. Nistala GJ, Wu K, Rao CV, Bhalerao KD. A modular positive feedback-based gene amplifier. J Biol Eng. 2010;4:4.

32. Anderson JC, Clarke EJ, Arkin AP, Voigt CA. Environmentally controlled invasion of cancer cells by engineered bacteria. J Mol Biol. 2006;355:619-27.

33. Basu S, Mehreja R, Thiberge S, Chen MT, Weiss R. Spatiotemporal control of gene expression with pulse-generating networks. Proc Natl Acad Sci U S A. 2004:101:6355-60.

34. Basu S, Gerchman Y, Collins CH, Arnold FH, Weiss R. A synthetic multicellular system for programmed pattern formation. Nature. 2005;434:1130-4.

35. Xiao Y, Liu Q, Chen H, Zhang YX. A stable plasmid system for heterologous antigen expression in attenuated Vibrio anguillarum. Vaccine. 2011;29:6986-93

36. Kaplan HB, Greenberg E. Diffusion of autoinducer is involved in regulation of the Vibrio fischeri luminescence system. J Bacteriol. 1985;163:1210-4.

37. Zhou LY, Wang XH, Liu Q, Wang QY, Zhao Y, Zhang YX. A novel multivalent vaccine based on secretary antigen-delivery induces protective immunity against Vibrio anguillarum and Aeromonas hydrophila. J Biotechnol. 2010;146:25-30 
38. Xiao JF, Chen T, Liu B, Yang WZ, Wang QY, Qu J, et al. Edwardsiella tarda mutant disrupted in type III secretion system and chorismic acid synthesis and cured of a plasmid as a live attenuated vaccine in turbot. Fish Shellfish Immunol. 2013:35:632-41.

39. Choudhary S, Schmidt-Dannert C. Applications of quorum sensing in biotechnology. Appl Microbiol Biotechnol. 2010;86:1267-79.

40. Wang S, Li Y, Shi H, Sun W, Roland KL, Curtiss III R. Comparison of a regulated delayed antigen synthesis system with in vivo-inducible promoters for antigen delivery by live attenuated Salmonella vaccines. Infect Immun. 2011;79:937-49.

\section{Submit your next manuscript to BioMed Central} and take full advantage of:

- Convenient online submission

- Thorough peer review

- No space constraints or color figure charges

- Immediate publication on acceptance

- Inclusion in PubMed, CAS, Scopus and Google Scholar

- Research which is freely available for redistribution 